\title{
Evolutionary Parameter Estimation for a Theory of Planned Behaviour Microsimulation of Alcohol Consumption Dynamics in an English Birth Cohort 2003 to 2010
}

\author{
Robin C. Purshouse \\ Department of Automatic \\ Control \& Systems \\ Engineering \\ University of Sheffield \\ Sheffield, UK \\ r.purshouse@sheffield.ac.uk \\ Daniel Moyo \\ Department of Automatic \\ Control \& Systems \\ Engineering \\ University of Sheffield \\ Sheffield, UK \\ d.moyo@sheffield.ac.uk
}

Abdallah K. Ally

School of Health \& Related

Research

University of Sheffield

Sheffield, UK

a.k.ally@sheffield.ac.uk

\author{
Alan Brennan \\ School of Health \& Related \\ Research \\ University of Sheffield \\ Sheffield, UK \\ a.brennan@sheffield.ac.uk
}

\author{
Paul Norman \\ Department of Psychology \\ University of Sheffield \\ Sheffield, UK \\ p.norman@sheffield.ac.uk
}

\begin{abstract}
This paper presents a new real-world application of evolutionary computation: identifying parameterisations of a theory-driven model that can reproduce alcohol consumption dynamics observed in a population over time. Population alcohol consumption is a complex system, with multiple interactions between economic and social factors and drinking behaviours, the nature and importance of which are not well-understood. Prediction of time trends in consumption is therefore difficult, but essential for robust estimation of future changes in health-related consequences of drinking and for appraising the impact of interventions aimed at changing alcohol use in society. The paper describes a microsimulation approach in which an attitude-behaviour model, Theory of Planned Behaviour, is used to describe the frequency of drinking by individuals. Consumption dynamics in the simulation are driven by changes in the social roles of individuals over time (parenthood, partnership, and paid labour). An evolutionary optimizer is used to identify parameterisations of the Theory that can describe the observed changes in drinking frequency. Niching is incorporated to enable multiple possible parameterisations to be identified, each of which can accurately recreate history but potentially encode quite different future trends. The approach is demonstrated using evidence from the 1979-1985 birth cohort in England between 2003 and 2010 .
\end{abstract}

\section{Categories and Subject Descriptors}

I.6 [Simulation and Modeling]: Model Validation and Analysis

\section{General Terms}

Experimentation

\section{Keywords}

Simulation optimization; Social science; Genetic algorithms; Multiple solutions / niching

\section{INTRODUCTION}

Alcohol consumption is regarded as a major public health issue internationally, with alcohol recognised as a risk factor across a wide range of diseases, including neuropsychiatric disorders, gastrointestinal disease and cancer, and both intentional and unintentional injuries. The World Health Organization estimates that $4.5 \%$ of the global burden of disease and injury in 2004 was attributable to alcohol [31]. As a result, policies that aim to reduce the harms caused by alcohol are under consideration in many jurisdictions, including population-level interventions to reduce the affordability of alcohol to consumers (such as introducing a floor price for alcoholic beverages), e.g. [28]. With an increasing societal demand for such interventions to be evidence-based, policymakers are increasingly commissioning mathematical models that aim to appraise the likely impact of policy options across a range of criteria, drawing on input assumptions based on relevant data - see, for example, [22].

The current state-of-the-art methods for model-based appraisal are grounded in neoclassical economics, where the effects of an intervention are estimated ceteris paribus - in other words, a static approach where the dynamics of the system are ignored [24]. However, since alcohol consumption in a society is a complex system driven by many causal 


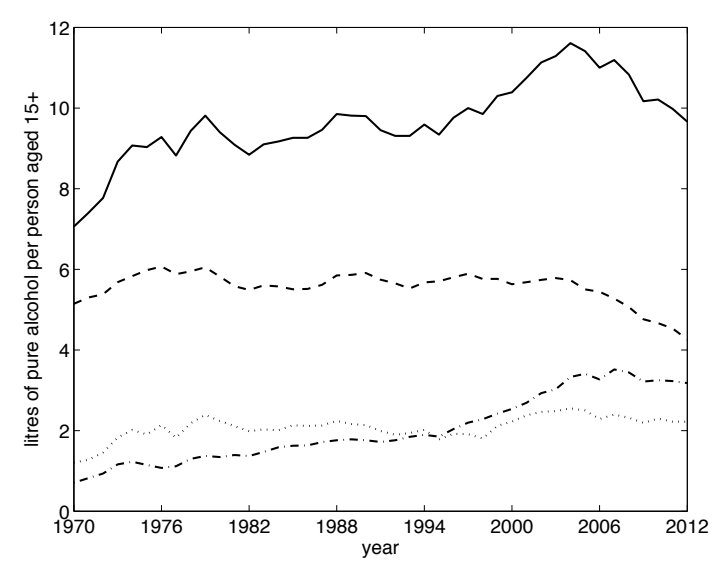

Figure 1: Alcohol consumption in the UK (litres of ethanol per person aged 15 and over): - all alcohol; - - - beer and cider; - . - wine; $\cdots$ spirit and ready-to-drink [4].

factors [17, 25, 26], patterns of drinking in a population tend not to be in stasis, but rather to be varying in ways that are not fully understandable or predictable (see Figure 1, showing 42 years of change in estimated UK per capita consumption).

In this setting, the ceteris paribus assumptions of neoclassical models may lead to incorrect estimates for intervention effects, and also make validation of the models on observed data exceptionally difficult to perform. Such concerns have foundation, but have become increasingly distorted by ideologically-driven libertarian critics of population-level interventions, who now question the very basis of using models for prediction of policy effects, claiming for example, "There is no shame in saying that we simply do not know." $[10$, p. 12], despite the portfolio of evidence available for analysis.

To overcome these unconstructive yet influential critics of model-based appraisal, we need new methods that account for alcohol as a dynamic factor in a complex system, that are underpinned by theory that supports a causal argument, that can reproduce historical data and that provide credible intervals around predictions of future impacts. This is an agenda that has been recognised by sociologists for over 50 years, including the early cybernetics work on drivers of change (morphogenesis) and stasis (morphostasis) in social systems by Walter Buckley [5] and, more recently, the vision of British sociologist David Byrne:

"... to identify historical dynamics of particular socio-spatial systems - with models of how such systems might have been different at this point in time - coupled with forward projections ... This is a new kind of 'social engineering science', a rational programme not of assertion based on absolute prediction, but of social action based on specification of the multiple but not limitless range of ...options ... We have the possibility of qualitative representations of alternative futures." [6, p. 166-7]

Despite the apparent strong potential of complex systems methods for modelling alcohol consumption dynamics, disappointingly little progress has been made to date. The few

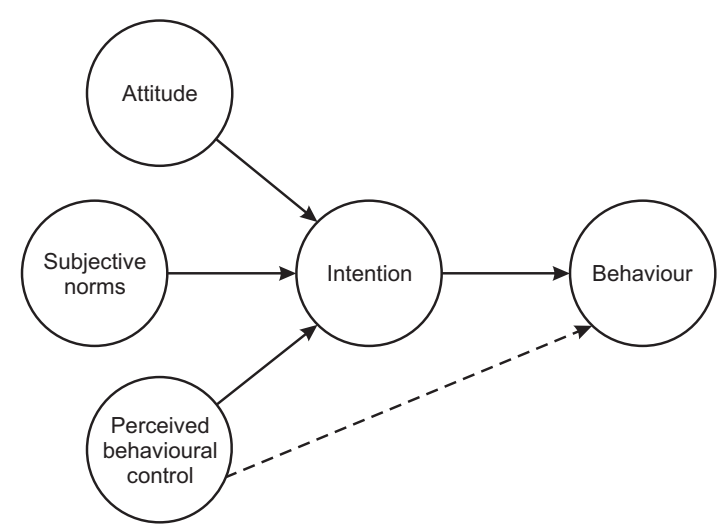

Figure 2: Schematic of the Theory of Planned Behaviour: - link; - - potential link.

studies available either lack an appropriate theoretical basis, lack any empirical validation of results, or suffer from both these issues [12, 13, 15].

In this paper, we set out a new approach to dynamic complex systems modelling of alcohol consumption that is underpinned by theory, is able to reproduce historical observations, and includes uncertainty estimates in its predictions.

The remainder of the paper is organised as follows. In Section 2, the proposed complex systems modelling methodology for the purposes of describing and predicting drinking behaviours over time is introduced. Results are shown in Section 3 for an exemplar population sub-group in England. Section 4 concludes with a comparison of the findings to existing knowledge, a discussion of strengths and limitations of the proposed methodology, and research directions.

\section{METHOD}

\subsection{The Theory of Planned Behaviour}

The theory selected for investigation in the study is the Theory of Planned Behaviour (TPB), which is a well established social psychological model [2]. The TPB has been previously used with some success in the prediction of alcohol consumption behaviours in field studies [7]. As shown in Figure 2, the theory posits that behaviour (for example, consuming alcohol or drinking to intoxication) is driven by the immediate antecedent intention (i.e. decision) to perform that behaviour, and that the intention is itself driven by three psychological factors:

- Attitude - The extent to which an individual positively values the behaviour to be predicted.

- Subjective norms - An individual's perception over whether other people who are important to her would approve or disapprove of her performing the behaviour.

- Perceived behavioural control (PBC) - An individual's perception of her level of control over engaging in the behaviour and her perceived ability to perform the behaviour. If an individual has accurate perceptions of actual control, PBC may also directly affect behaviour.

To construct a quantitative model of the TPB process, population weightings are assigned to indicate the relative 
importance of attitude, subjective norms and perceived behavioural control. When combined with individual data on these factors, the weightings produce an overall intention score. The score is compared to some threshold to determine whether or not the individual is predicted to perform the behaviour. In our implementation, we define behaviour as frequency of drinking an alcoholic drink on an ordinal categorical scale $y \in\left\{y_{1}=\right.$ once a month; $y_{2}=2-3$ times a month; $y_{3}=$ once or twice a week; $y_{4}=$ most days $\}$. We then determine the probability that an individual $i$ will engage in drinking category $j$ according to a cumulative logit model [1]:

$$
\operatorname{logit}\left[P\left(Y \leq j \mid \mathbf{x}_{i}\right)\right]=\alpha_{j}-\boldsymbol{\beta}^{\mathrm{T}} \mathbf{x}_{i}
$$

where $\boldsymbol{\beta}$ is a set of population weightings relating to both TPB variables and demographic variables. The values of these exogenous variables for each individual are denoted by $\mathbf{x}_{i}$. The $\alpha_{j}$ in (1) are intercepts that determine the logarithmic odds of an individual drinking at a level less than or equal to $j$ for the reference case $\mathbf{x}=0$.

The set of exogenous variables used in the model is constrained by data availability. In the study, we were restricted to secondary analysis of existing datasets; the most promising we found that had TPB-like measures together with an indication of drinking was the Offending, Crime and Justice Survey (OCJS) from 2003 [18]. This is a cross-sectional survey of the UK population aged 10-65 with a sample size of $6,892^{1}$. In our study, we restricted the analysis to persons aged 18 and over in England. We used the following data from OCJS to populate $\mathbf{x}$ for a baseline year in the model:

Attitude: The survey invites respondents to indicate the level to which they agree or disagree with the following statements related to alcohol consumption: "Drinking alcohol makes me feel relaxed"; "Drinking makes me forget my problems"; "Drinking makes me feel more friendly and outgoing"; "I drink to get drunk".

Subjective norms: Specific questions on an individual's perceptions of drinking norms are not included in the survey. Therefore, we use a question that invites respondents to indicate the types of people they usually drink with i.e. "by self, parents, spouse/partner, girl/boyfriend, friends, siblings, other relatives, work colleagues, others" - and count the number of types selected by the respondent as a proxy measure of perceived social pressure to drink.

Perceived behavioural control: Again, specific questions on perceived control are not included in the survey. We use a question that invites respondents to indicate the types of location where they usually drink - i.e. "own home, other's home, pub/bar, nightclub/disco, restaurant, party, youth/community club, street/park/outdoors, elsewhere" and count the number of types selected by the respondent as a proxy measure of facilitating factors that may increase perceptions of control. We also include a count of the number of social roles held by the individual, in terms of parenthood (having a dependent child aged under 18), partnership (cohabitation within a relationship) and paid labour (holding a salaried job).

\footnotetext{
${ }^{1}$ OCJS also has a major longitudinal component from 20032006 , but this is limited to persons aged under 25, with no repeated measures on alcohol consumption or attitudes to drinking.
}

Demographics: Data is available on the sex and age of the respondent and the highest level of education attained. Responses to the latter are mapped on to the UK National Qualifications Framework (NQF) [21].

\subsection{Microsimulation of the Theory}

The TPB model is embedded within a wider microsimulation which aims to describe the dynamics of drinking in a population over time. The microsimulation is an agentbased model (ABM) designed according to object-oriented principles. In the ABM, agent objects represent 1,845 individual respondents to the OCJS survey and encode both TPB properties and demographics (i.e. an agent $i$ encodes properties $\mathbf{x}_{i}$ ). Agent frequency of drinking is then evaluated using (1) to identify probabilities of being in each possible drinking state. The actual drinking state $y$ is then selected via a uniform random number generator.

Dynamics are introduced into the microsimulation by allowing certain agent properties to vary over time - specifically, in this first version of the ABM, age and the three social roles (parenthood, partnership and paid labour). An annual update cycle, $t$, is chosen, such that (1) is extended to a dynamic form:

$$
\operatorname{logit}\left[P\left(Y(t) \leq j \mid \mathbf{x}_{i}(t)\right)\right]=\alpha_{j}-\boldsymbol{\beta}^{\mathrm{T}} \mathbf{x}_{i}(t)
$$

Social role trajectories are defined for each agent as exogenous inputs to the ABM. Trajectories are extracted from a separate longitudinal study, the British Household Panel Survey (BHPS) which has tracked the lives of over 10,000 individuals in Great Britain since 1991 [29]. Trajectories for parenthood, partnership and paid labour are constructed for 7,309 English individuals who were aged 18-65 in 2003, for the period 2003-2009 (the latest available data). A simple trend analysis is used to estimate trajectory development for 2010. At initialisation, agents in the ABM are matched to samples from the BHPS, enabling social role trajectories to be imputed. Agents are matched using sex, age, education and social role states at baseline.

The microsimulation is implemented in Python v2.7.5. Code is available from the authors.

\subsection{Evolutionary Parameter Estimation}

The TPB weights, $\boldsymbol{\beta}$, and thresholds, $\boldsymbol{\alpha}$, are estimated via an optimization process. In addition to identifying parameterisations that can reproduce observed time trends in the frequency of drinking, the optimizer is designed to find diversity in such parameterisations.

An evolutionary algorithm (EA) has been selected as the optimization engine since it can handle non-differentiable cost functions, provides an element of robustness to the stochastic nature of the ABM, and can be extended to include mechanisms that promote a search for diverse, high quality, solutions. The overall structure of the EA is given by:

$$
\mathcal{P}[g+1]=s_{s}\left(v\left(s_{v}(\mathcal{P}[g])\right), \mathcal{P}[g]\right)
$$

where $\mathcal{P}[g]$ is the population of candidate solutions (i.e. a collection of particular configurations of $\boldsymbol{\alpha}$ and $\boldsymbol{\beta}$ ) at iteration $g$ of the algorithm, $s_{v}$ is the selection-for-variation operator, $v$ is the variation process, and $s_{s}$ is the selectionfor-survival operator. The components of the EA are described in more detail below. 
Table 1: EA parameter settings

\begin{tabular}{ll}
\hline Parameter & Setting \\
\hline \hline Population size, $N$ & 100 \\
Generations, $G$ & 200 \\
SBX crossover probability, $p_{c}$ & 1 \\
SBX exchange probability, $p_{e}$ & 0.5 \\
SBX crossover strength, $\eta_{c}$ & 15 \\
Polynomial mutation probability, $p_{m}$ & $1 / 13$ \\
Polynomial mutation strength, $\eta_{m}$ & 20 \\
\hline
\end{tabular}

\subsubsection{Representation}

Each candidate solution, $\mathbf{p}$, represents a particular choice of TPB parameters $-\mathbf{p}=\left[\beta_{a 1}, \beta_{a 2}, \beta_{a 3}, \beta_{a 4}, \beta_{n 1}, \beta_{p 1}, \beta_{p 2}\right.$, $\left.\beta_{d 1}, \beta_{d 2}, \beta_{d 3}, \alpha_{1}, \alpha_{2}, \alpha_{3}\right]$ - where the definition of each of the 13 components in the chromosome is given in Table 2. Each component is represented by a real number within the range $[-10,+10]$.

\subsubsection{Cost Function}

A time series of drinking frequency for 2003-2010 is extracted from a repeated cross-sectional survey, the Health Survey for England (HSE), which has included a variety of questions on drinking for individual respondents aged 16 and over in England since the inception of the survey in 1991 [20]. Sample size varies between 4,645 and 15,102, depending on year, over the analysis period. The time series is expressed as a sequence of counts, $c_{\text {actual }, j}(t)$, of the number of HSE respondents in each drinking state $y_{j}$ at time $t$.

To construct a cost function for a candidate solution, the HSE-derived time series $\mathbf{c}_{\text {actual }}$ is compared to the estimates of the same time series coming from the microsimulation when parameterised with p. Here, the sequence of counts $c_{\text {model }, j}(t)$ relate to the number of agents in each drinking state $y_{j}$ over time $t$. For each year, the HSE and ABM counts are formulated as a contingency table, $\mathbf{C}(\mathbf{p}, t)$, as shown in (4). The similarity of the two distributions is then assessed using a chi-square test of independence, where the output used in the cost function is the chi-square test statistic, $\chi^{2}$. The total cost for the candidate solution is obtained by taking the mean of the test results for every year of the time series, as shown in (5), where $T=7$ is the total number of years of data.

$$
\begin{gathered}
\mathbf{C}(\mathbf{p}, t)=\left[\begin{array}{ccc}
c_{\text {actual }, j=1}(t) & \ldots & c_{\text {actual }, j=J}(t) \\
c_{\operatorname{model}(\mathbf{p}), j=1}(t) & \ldots & c_{\operatorname{model}(\mathbf{p}), j=J}(t)
\end{array}\right] \\
e(\mathbf{p})=\frac{1}{T} \sum_{t=1}^{T} \chi^{2}[\mathbf{C}(\mathbf{p}, t)]
\end{gathered}
$$

Since smaller values of $\chi^{2}$ indicate better matches between the ABM results and the observed HSE data, the aim of the optimization process is to find solutions that minimize the total error, $e$, in (5).

\subsubsection{Selection-for-variation}

To convert cost function results into fitness values, the population is ranked according to $e$ and fitnesses are assigned by interpolating exponentially between 1 (for the worst rank) and 10 (for the best rank). In order to promote diversity across the range of good parameterisations sought, we incorporate a well-known niching strategy - fitness sharing - into the selection process [14]. Since the performance of fitness sharing is known to be sensitive to the choice of its niche size parameter, we make use of an existing adaptive approach for setting this parameter based on guidelines for the analogous Epanechnikov kernel density estimator [11].

Once fitness values have been calculated, selection is performed using stochastic universal sampling, which is effective in minimizing undesirable sampling variation and biases [3].

\subsubsection{Variation}

Since the chosen representation is based entirely on real numbers, it is natural to adopt real-parameter variation operators for the study. We first apply a recombination operator and then a mutation operator to the candidate solutions selected for variation:

Simulated binary crossover (SBX): uses two selected solutions to produce two new solutions, drawing new values from a probability distribution [8]. The scale of the distribution is governed by the distance between the parent solutions; the shape is governed by a parameter $\eta_{c}$. The decision to apply SBX to parent solutions is made with probability $p_{c}$. Actual crossover operations are applied independently to each of the 13 components of the chromosome with probability 0.5. The resulting values for the components are exchanged between the new solutions with probability $p_{e}$.

Polynomial mutation: uses one selected solution to produce one new solution [9]. The decision to apply the operator is made at component-level with probability $p_{m}$. A new value is produced from a probability distribution centred over the parent value. The scale of mutation is determined by a parameter $\eta_{m}$.

\subsubsection{Selection-for-survival}

The use of fitness sharing in combination with quite disruptive variation operators indicates that convergence of the algorithm might be slow and this was confirmed through early empirical testing. To increase selective pressure, we therefore adopt an elitist approach for updating the population from the pool of parent, $\mu$, and child, $\lambda$, solutions. Fitness is calculated on the pool $(\mu+\lambda)$ according to Section 2.3.3 and truncation selection is used to select the fittest $N$ solutions to be carried forward to the next generation.

A summary of the EA configuration is given in Table 1. The algorithm was implemented in Matlab 7.7.0 (using a 64-bit floating-point encoding for the real number representation). Code is available from the authors.

\section{RESULTS}

In this paper we demonstrate modelling results for the 1979-1985 birth cohort in England (encoded by 220 agents), who were aged 18 to 24 in 2003, in terms of population-level frequency of drinking. In Section 3.1, we show results of the model fitting process over years 2003 to 2009. In Section 3.2, we show model predictions for 2010 and compare these to the observed drinking frequencies for that year.

\subsection{Model Fitting}

The convergence profile achieved by the EA is shown in Figure 3. Model fit in the early stages of the optimization process is very poor but rapid progress is made toward a level where the observed and modelled distributions should begin to look similar (a conventional chi-square test for in- 


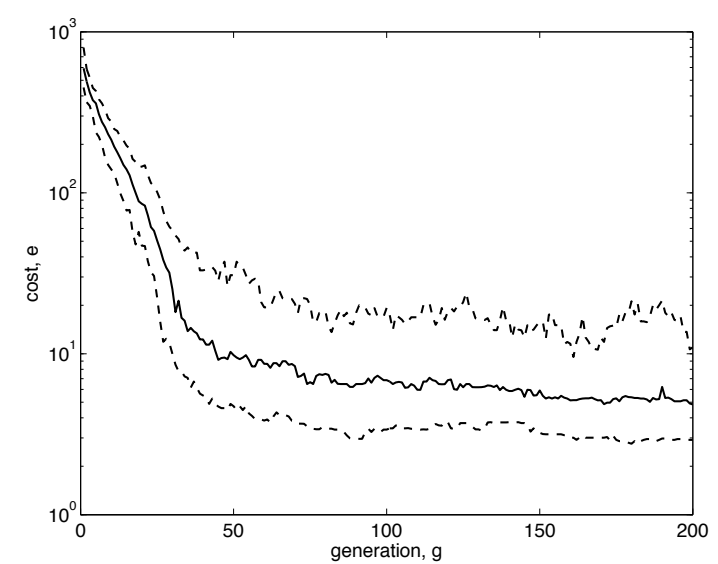

Figure 3: Convergence profile of the EA: showing 10\% (broken), 50\% (unbroken) and 90\% (broken) deciles for $e(\mathbf{p} \in \mathcal{P}(g))$.

dependence at the $1 \%$ level would accept the null hypothesis for $\left.\chi^{2} \preccurlyeq 11.3\right)$. Convergence continues at a slower rate from $g \approx 50$, with the effect of fitness sharing at maintaining diversity in the population apparent in the wide spread of performance even at later stages of the process.

In the remainder of this section, we focus on the three best candidate models (ranked by cost) returned from the optimizer at generation 200. The time series produced by each of these models is shown in Figure 4, alongside the observed time series from HSE. The main trend in frequency of drinking discernible from the HSE is the rise in the prevalence of "most days" drinking $\left(y_{4}\right)$ to a peak of $20.4 \%$ in 2005 , followed by a sustained reduction in prevalence to a low of $9.2 \%$ by 2009 . The opposite trend occurred in the neighbouring category of "once or twice per week" drinking, with relatively static prevalences for the two categories of less frequent drinking. By inspection, all three models seem able to reproduce the observed dynamics reasonably well, particularly over the first half of the simulation window. However, none of the models capture the peak in "most days" drinking in 2005. Also, whilst Model 3 is best able to capture the decay in "most days" drinking up to 2009, it simultaneously over-predicts the prevalence in $y_{2}$ and under-predicts the prevalence in $y_{3}$ between 2007 and 2009.

The chromosomes and costs associated with the three best models are shown in Table 2. Whilst the models have similar costs $(2.084,2.322$ and 2.540), they encompass quite a diverse range of cumulative logit model parameterisations, as encouraged by the niching strategy.

TPB parameters: In terms of attitudes, drinking for relaxation and social purposes $\left(\beta_{a 1}\right.$ and $\left.\beta_{a 3}\right)$ are consistently associated with increased odds of more frequent consumption across the models. The relative ability of these attitudes to change behaviours differs between models (e.g. for $\beta_{a 1}$, a unit change in response will elicit the greatest change in drinking probabilities in Model 3 and the smallest change in Model 1). However there are fundamental differences between the models when it comes to the impact of using alcohol as a coping strategy $\left(\beta_{a 3}\right)$ or for hedonistic purposes $\left(\beta_{a 4}\right)$. In terms of subjective norms $\left(\beta_{n 1}\right)$, Model 1 and Model 2 associate increasing numbers of drinking partner types with increased drinking frequency, whilst Model 3 en-
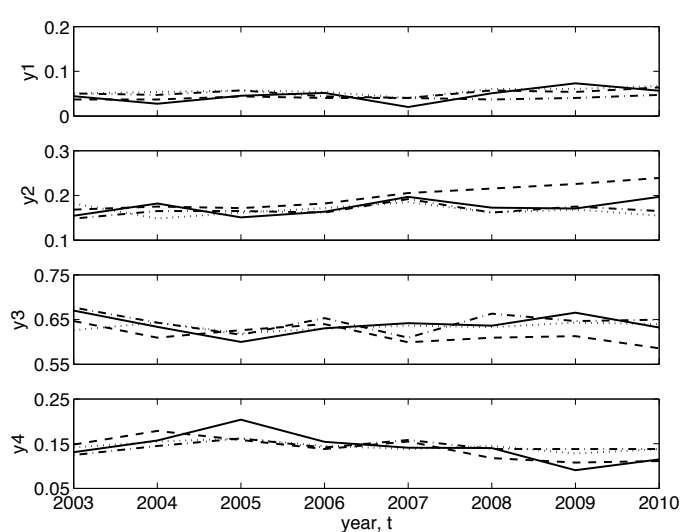

Figure 4: Time series for $\mathbf{y}(t)$ prevalence in the 1979-1985 birth cohort, covering optimization window 2003-2009 and validation year 2010: - HSE observations; $\cdots$ Model 1; - - - Model 2; - - - Model 3.

codes the opposite. In terms of perceived behavioural control, only Model 3 indicates a positive association between number of drinking location types $\left(\beta_{p 2}\right)$ and drinking frequency $(+2.116)$, which is arguably the direction of effect with the most face validity. All three models associate an increase in the number of social roles $\left(\beta_{p 2}\right)$ with decreased odds of engaging in more frequent drinking - with Model 3 placing the greatest emphasis on this parameter (-3.321).

Demographic parameters: Sex of respondent $\left(\beta_{d 1}\right)$ is identified as a key driver of drinking frequency across the three models. Whilst this result will not surprise many in the alcohol research community, the direction of effect in Model 2 (+6.015 for females) arguably lacks face validity since most of the evidence for alcohol participation levels in Western countries shows a positive association for males rather than females [31]. Increased age of respondent $\left(\beta_{d 2}\right)$ is associated with a small increase in odds of more frequent drinking across the models. In terms of highest educational qualification level gained, Models 1 and 3 suggest only very small (and positive) effects, whilst Model 2 suggests a strong negative association between higher educational qualifications and drinking frequency (-3.756).

Overall, despite having the loosest fit to the totality of historical data of the three models considered, Model 3 arguably has the most face validity in terms of the size and strength of the cumulative logit model coefficients. This model was also best able to reproduce the observed trend in "most days" drinking, but mistakenly over-emphasised changes across other drinking categories.

\subsection{Prediction}

In this section we consider the ability of the models fitted to data for 2003-2009 as predictors of macro-level drinking frequencies in 2010, denoted as $\hat{\mathbf{y}}(2010)$. Rather than focus on individual models and the face validity of their parameterisations, here we consider the final population of the EA as a whole and use it to generate a probability density estimate around the predicted variable, $\mathbf{f}(\hat{\mathbf{y}})$. Each candidate solution in $\mathcal{P}(200)$ has its own prediction for $\hat{\mathbf{y}}(2010)$. Focusing on the marginal distributions of $\mathbf{f}(\hat{\mathbf{y}})$, a histogram is constructed of the population of predictions. The contribution of each prediction to the probability density of the 
Table 2: Theory of Planned Behaviour parameter estimates (values to 3 d.p.) for a microsimulation-embedded cumulative logit model (1) of drinking frequency $y=\{$ once a month;2-3 times a month;once or twice a week;most days $\}$ for the 18-24 year old 2003 cohort in England

\begin{tabular}{|c|c|c|c|c|c|}
\hline Type & Description & Parameter & Model 1 & Model 2 & Model 3 \\
\hline \multirow{6}{*}{ Attitude } & "Drinking alcohol makes me feel relaxed & 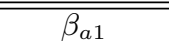 & +0.321 & +2.691 & +3.584 \\
\hline & Drinking makes me forget my problems & $\beta_{a 2}$ & -2.903 & -2.724 & +1.710 \\
\hline & Drinking makes me feel more friendly and outgoing & $\beta_{a 3}$ & +0.445 & +1.847 & +0.710 \\
\hline & I drink to get drunk & $\beta_{a 4}$ & +4.882 & -1.282 & -4.140 \\
\hline & Coding for all attitudes: & & & & \\
\hline & $\begin{array}{l}(0=\text { disagree strongly, } 1=\text { disagree slightly, } \\
2=\text { agree slightly, } 3=\text { agree strongly) }\end{array}$ & & & & \\
\hline \multirow{2}{*}{ Subjective norms } & Count of drinking partner types & $\beta_{n 1}$ & +1.945 & +3.372 & -1.967 \\
\hline & $(0=1,1=2, \ldots, 8=9)$ & & & & \\
\hline \multirow{2}{*}{$\begin{array}{l}\text { Perceived } \\
\text { behavioural control }\end{array}$} & $\begin{array}{l}\text { Count of drinking place types } \\
(0=1,1=2, \ldots, 8=9)\end{array}$ & $\beta_{p 1}$ & -0.739 & -1.480 & +2.116 \\
\hline & $\begin{array}{l}\text { Number of social roles } \\
(0,1, \ldots, 3)\end{array}$ & $\beta_{p 2}$ & -0.779 & -0.262 & -3.321 \\
\hline \multirow{5}{*}{ Demographics } & $\begin{array}{l}\text { Sex } \\
(0=\text { male }, 1=f \text { emale })\end{array}$ & $\beta_{d 1}$ & -7.744 & +6.015 & -5.940 \\
\hline & Age group & $\beta_{d 2}$ & +0.220 & +0.095 & +0.039 \\
\hline & $(0=18-24,1=25-34,2=35-44,3=44-54,4=55+)$ & & & & \\
\hline & Highest educational qualification & $\beta_{d 3}$ & +0.026 & -3.756 & +0.040 \\
\hline & $\begin{array}{l}\text { NFQ scales: }(0=\text { entry/other,1=Level } 1 \text {, } \\
\text { 2=Level 2,3=Levels } 3-5,4=\text { Levels } 6+)\end{array}$ & & & & \\
\hline \multirow{4}{*}{ logit intercepts } & log-odds of $y=$ once a month $\mid \mathbf{x}=0$ & $\alpha_{1}$ & -9.929 & -9.934 & -9.951 \\
\hline & log-odds of $y \leq 2-3$ times a month $\mid \mathbf{x}=0$ & $\alpha_{2}$ & -4.060 & -4.106 & -2.827 \\
\hline & $\log$-odds of $y \leq$ once or twice per week $\mid \mathbf{x}=0$ & $\alpha_{3}$ & +9.292 & +9.894 & +9.373 \\
\hline & & Cost & 2.084 & 2.322 & 2.540 \\
\hline
\end{tabular}

histogram is weighted according to the ability of that model to recreate past events. Specifically, the weighting is taken as the p-value for independence between observed and modelled distributions using an average $\chi^{2}$ for the time series - i.e. using Equation 5. Results are plotted in Figure 5, where the histogram has been smoothed using a normal kernel function.

The HSE observations for 2010 can be seen to lie well within the intervals predicted by the ensemble of models, and quite close to the median estimates. In particular, for "most days" drinking, the ensemble ascribes an $86 \%$ likelihood to an arrest in the trend of decreasing prevalence observed since 2005 - an outcome that did in fact occur in the HSE data for 2010. This is clearly a very different modelling estimate to what would result from a simple trend line fitted to HSE "most days" data for period 2005 to 2010. Specifically, whilst the observed prevalence increases from $y_{4}(2009)=9.15 \%$ to $y_{4}(2010)=11.46 \%$, linear and exponential trend lines predict continuing decreases in prevalence to $\hat{y}_{4}(2010)=7.47 \%$ and $\hat{y}_{4}(2010)=8.52 \%$ respectively.

\section{CONCLUSION}

\subsection{Findings}

This study is the first of its kind to build a dynamic, quantitative, model of psychological theory that aims to explain and predict change and stasis in patterns of population-level alcohol consumption. The use of a theory-driven approach allows individual models to be analysed for face validity, whilst the search for an ensemble of diverse models that can replicate history enables uncertainty intervals to be estimated for the predicted outcome.
In this paper, the proposed methodology has been demonstrated by using TPB concepts to model the dynamics of drinking frequency in the 1979-1985 birth cohort in England between 2003 and 2010. The optimizer was able to find diverse parameterisations of the model that could reproduce historical data, with the results used to generate uncertainty estimates for a one-step-ahead prediction of population drinking frequencies.

In Table 2, the finding of larger attitude and subjective norm weightings, in relation to perceived behavioural control weightings, is broadly in-line with existing TPB studies of alcohol consumption [7]. In TPB, the explanatory power of the psychological components is hypothesised to render the demographics parameters unimportant; however, in the model, sex remains an important driver of consumption behaviour. This is likely to be because, in a conventional TPB field study, the research team can carefully select the instruments used to measure the psychological components relating to the behaviour under investigation, whereas here we are limited to existing datasets with only proxy measures for these components.

The finding of a negative relationship between number of social roles and frequency of drinking in Table 2 is broadly in-line with recent empirical findings. For example, a study of 10 Western countries found that increasing numbers of social roles were associated with reduced odds of engagement in heavy drinking for both males and females, when observations were grouped across all 10 countries [19]. However our findings are somewhat stronger than those in [19] when considering that study's UK-specific analysis, wherein a statistically significant relationship was found only in males holding three roles (in comparison to no roles). 

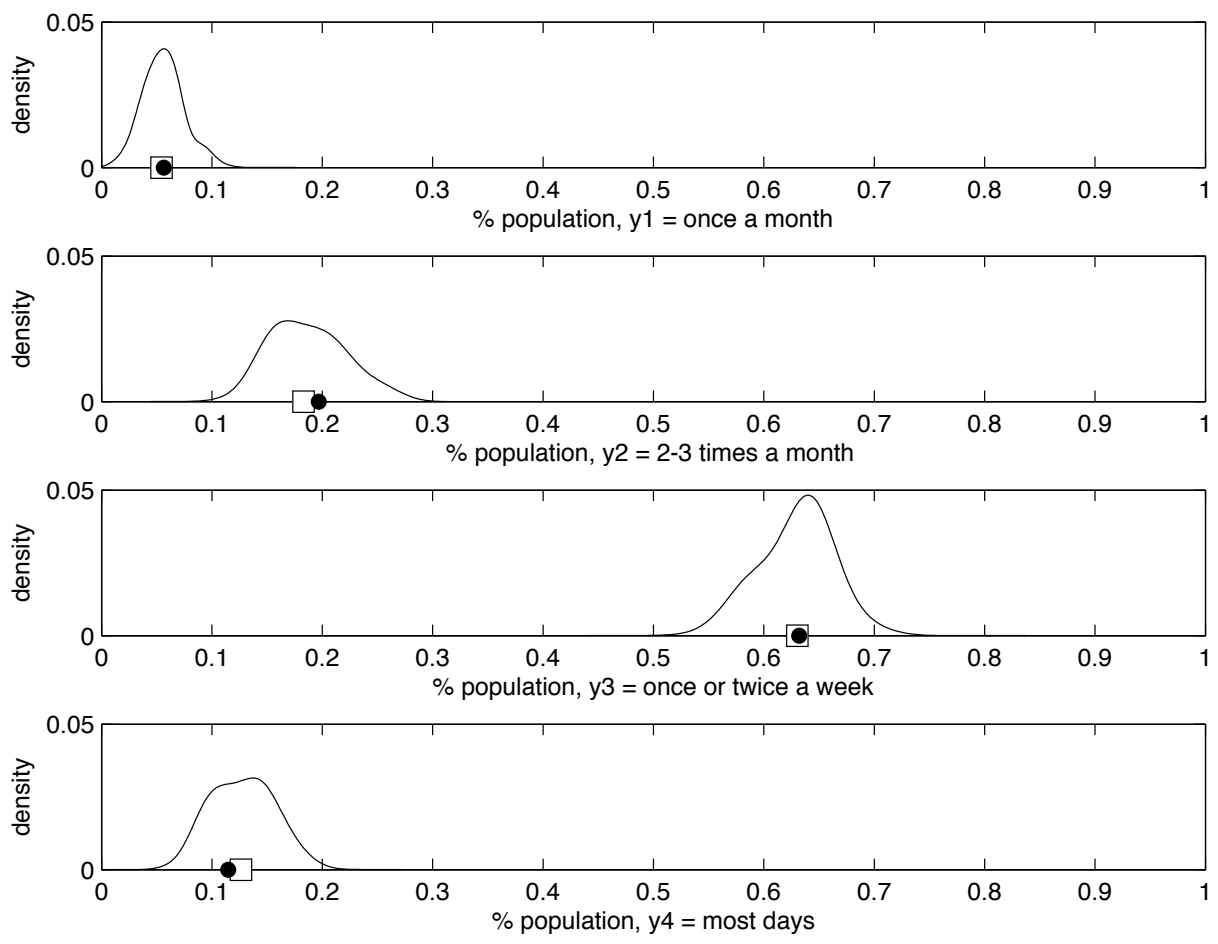

Figure 5: Marginal probability density estimates for the prediction of $\mathbf{y}(2010)$ based on $\mathcal{P}(200)$. Median predictions are shown as squares $(\square)$. Observations from HSE 2010 are indicated by the filled circles $(\bullet)$.

\subsection{Limitations and Future Work}

Whilst the method has generated highly promising results for one population sub-group in one country, these findings must be regarded as indicative of proof-of-principle at this stage. Further work is needed to verify if the method is reproducible on other population sub-groups both in England and in other jurisdictions. Furthermore, as yet we have taken little account of the potential effects of stochasticity, both in the ABM and the EA, on the robustness of the proposed modelling methodology. A full statistical robustness analysis is needed, with countermeasures introduced to improve robustness if this is found to be necessary. It will also be instructive to compare the method with more traditional regression approaches, as well as state-of-the-art model calibration methods, including evolutionary [27] and Bayesian [30] techniques.

From the perspective of analytical sociology [16], the generative account of how micro-level dynamics produce macrolevel outcomes (i.e. the TPB microsimulation of drinking frequency) is incomplete since it does not include the social interactions that occur between individuals. Such interactions may be important for capturing the dynamics of, for example, attitude change, that may prove important for both explanation and prediction of population drinking trends. Future work will seek to introduce interaction effects into the microsimulation, e.g. adaptive models of attitudes based on either explicit or proxy measures of social connectedness between individuals.

A further limitation of the microsimulation is that it is not entirely endogenous - specifically, social role trajectories are required as exogenous inputs to the model, which in themselves may not be straightforward to predict. However, the notion of a microsimulation or ABM being able to capture all of the actions and interactions necessary to explain and predict an empirically observed social phenomenon remains something of an ideal; pragmatically, exogenous inputs (e.g. network structures) are a short-term necessity.

Future work will also examine alternative measures of consumption to be predicted by the model. Frequency of intoxication is also available in the OCJS dataset, but the outcome of greatest utility would be mean grams per day of ethanol consumed because this measure can be used directly in the existing epidemiological models that relate drinking to chronic health harms [23]. Unfortunately mean consumption is not captured by OCJS and therefore imputation will be required. We view new or enhanced population surveys that integrate, in a theory-led way, psychological and social factors with alcohol consumption (and other behaviours relevant to public health) as a priority for future funding.

In summary, we believe that the proposed methodology has the potential to be transformative in the use of complex systems methods for model-based policy appraisal, specifically in terms of alcohol, but also more widely within public health, where achieving an understanding of the dynamics of macro-level behaviour change remains a core challenge.

\section{ACKNOWLEDGMENTS}

This work was funded by the UK Economic and Social Research Council under grant number ES/K001760/1.

The original data creators, depositors, or copyright holders, the funders of the data collections (when different), and the UK Data Archive bear no responsibility for analyses or interpretation of the data described in this report. The BHPS, HSE and OCJS are Crown Copyright. 


\section{REFERENCES}

[1] A. Agresti. Categorical Data Analysis. Wiley, New Jersey, 3rd edition, 2013.

[2] I. Ajzen. The theory of planned behavior. Organizational Behavior and Human Decision Processes, 50(2):179-211, 1991.

[3] J. E. Baker. Reducing bias and inefficiency in the selection algorithm. In Proceedings of the Second International Conference on Genetic Algorithms, pages 14-21, 1987.

[4] British Beer and Pub Association. Statistical Handbook 2013, 2013.

[5] W. Buckley. Sociology and Modern Systems Theory. Prentice Hall, New Jersey, 1967.

[6] D. S. Byrne. Complexity Theory and the Social Sciences: An Introduction. Routledge, Abingdon, 1998.

[7] R. Cooke, M. Dahdah, P. Norman, and D. P. French. How well does the theory of planned behaviour predict alcohol consumption intentions and behaviour? In BPS Division of Health Psychology Annual Conference 2013, 2013.

[8] K. Deb and R. B. Agrawal. Simulated binary crossover for continuous search space. Complex Systems, 9(2):115-148, 1995.

[9] K. Deb and M. Goyal. A combined genetic adaptive search GeneAS for engineering design. Computer Science and Informatics, 26(4):30-45, 1996.

[10] J. C. Duffy and C. Snowdon. The Minimal Evidence for Minimum Pricing: The Fatal Flaws in the Sheffield Alcohol Policy Model. ASI (Research) Ltd, London, 2012.

[11] C. M. Fonseca and P. J. Fleming. Multiobjective genetic algorithms made easy: Selection, sharing and mating restriction. In Proceedings of the First International Conference on Genetic Algorithms in Engineering Systems: Innovation and Applications, pages 42-52, 1995.

[12] L. A. Garrison and D. S. Babcock. Alcohol consumption amongst college students: an agent-based computational simulation. Complexity, 14(6):35-44, 2009.

[13] P. Giabbanelli and R. Crutzen. An agent-based social network model of binge drinking among Dutch adults. Journal of Artificial Societies and Social Simulation, 16(2):10, 2013.

[14] D. E. Goldberg and J. Richardson. Genetic algorithms with sharing for multimodal function optimization. In Proceedings of the Second International Conference on Genetic Algorithms, pages 41-49, 1987.

[15] D. M. Gorman, J. Mezic, I. Mezic, and P. J. Gruenewald. Agent-based modeling of drinking behavior: A preliminary model and potential applications to theory and practice. American Journal of Public Health, 96(11):2055-2060, 2006.

[16] P. Hedström. Dissecting the Social: On the Principles of Analytical Sociology. Cambridge University Press, Cambridge, 2005.

[17] H. D. Holder. Planning for alcohol-problem prevention through complex systems modelling: Results from
SimCom. Substance Use \& Misuse, 33(3):669-692, 1998.

[18] Home Office. Research, Development and Statistics Directorate. Offending Surveys and Research, National Centre for Social Research and BMRB. Social

Research. Offending, Crime and Justice Survey, 2003 [computer file]. 3rd Edition. Colchester, Essex: UK Data Archive [distributor], August 2008. SN: 5248.

[19] S. Kuntsche, R. A. Knibbe, and G. Gmel. Social roles and alcohol consumption: A study of 10 industrialised countries. Social Science \& Medicine, 68:1263-1270, 2009.

[20] National Centre for Social Research and University College London. Department of Epidemiology and Public Health. Health Survey for England, 2009 [computer file]. 2nd Edition. Colchester, Essex: UK Data Archive [distributor], July 2011. SN: 6732.

[21] Office of the Qualifications and Examinations Regulator. Qualifications can cross boundaries - a rough guide to comparing qualifications in the UK and Ireland. 3rd edition, 2011.

[22] R. C. Purshouse, P. S. Meier, A. Brennan, K. B. Taylor, and R. Rafia. Estimated effect of alcohol pricing policies on health and health economic outcomes in England: An epidemiological model. Lancet, 375:1355-64, 2010.

[23] J. Rehm, D. Bailunas, G. L. Borges, K. Graham, H. Irving, T. Kehoe, C. D. Parry, J. Patra, S. Popova, V. Poznyak, M. Roerecke, R. Room, A. V. Samokhvalov, and B. Taylor. The relation between different dimensions of alcohol consumption and burden of disease: An overview. Addiction, 105(5):817-843, 2010.

[24] G. Room. Complexity, Institutions and Public Policy Agile Decision-making in a Turbulent World. Edward Elgar, Cheltenham, 2011.

[25] R. Room, E. Osterberg, M. Ramstedt, and J. Rehm. Explaining change and stasis in alcohol consumption. Addiction Research and Theory, 17(6):562-576, 2009.

[26] J. Simpura. Trends in alcohol consumption and drinking patterns: Sociological and economic explanations and alcohol policies. Nordic Studies on Alcohol and Drugs, 18:3-13, 2001.

[27] F. Stonedahl and U. Wilensky. Evolutionary robustness checking in the Artificial Anasazi model. In Proceedings of the AAAI fall symposium on complex adaptive systems: resilience, robustness and evolvability, pages 120-129, 2010.

[28] The Scottish Government. Changing Scotland's Relationship with Alcohol: A Framework for Action. The Scottish Government, Edinburgh, 2009.

[29] University of Essex. Institute for Social and Economic Research. British Household Panel Survey: Waves 1-18, 1991-2009 [computer file]. 7th Edition. Colchester, Essex: UK Data Archive [distributor], July 2010. SN: 5151.

[30] I. Vernon, M. Goldstein, and R. G. Bower. Galaxy formation: a Bayesian uncertainty analysis. Bayesian Analysis, 5(4):619-670, 2010.

[31] World Health Organization. Global status report on alcohol and health. Technical report, WHO Press, 2011 . 